\title{
EFECTO DE LA DISPERSIÓN DE POLEN EN LA PRODUCCIÓN DE SEMILLA DE MAÍZ, EN TEXCOCO, MÉXICO ${ }^{1}$
}

\author{
Enrique Ortiz-Torres ${ }^{2}$, Aquiles Carballo-Carballo ${ }^{3}$, Abel Muñoz-Orozco ${ }^{3}$, Félix Valerio González-Cossio ${ }^{3}$
}

\section{RESUMEN}

Efecto de la dispersión de polen en la producción de semilla de maíz, en Texcoco, México. Con la finalidad de conocer cómo se dispersa el polen de maíz en un lote de producción de semillas con problemas de aislamiento y sus implicaciones en la calidad genética, se condujo un experimento en un lote de 189 por $42,5 \mathrm{~m}$ en el ciclo primaveraverano de 1992 en Texcoco, México. Se establecieron tres poblaciones de maíz contrastantes en la composición del endospermo: dos de tipo normal y una de tipo dulce. Las variedades de tipo normal estuvieron colocadas en tres surcos rodeando a la variedad dulce. Se evaluó la altura de planta y mazorca, floración femenina y masculina; además, el porcentaje de grano con endospermo normal, como indicativo del cruzamiento ocurrido en la variedad de endospermo dulce. Las condiciones que facilitaron el cruzamiento con las variedades de endospermo normal fueron la inexistencia de aislamiento por distancia y coincidencia de las floraciones. La dispersión de polen y subsecuente contaminación dentro del lote siguió una distribución normal; existieron variaciones en los niveles y distancias de contaminación debidas a la dirección de los vientos y en el vigor de las plantas contaminantes. La menor contaminación fue $1 \%$ que se presentó en el centro del lote. La ecuación de superficie de respuesta obtenida estimó que con 25 surcos borderos es posible obtener una calidad de semilla sin contaminación.

\begin{abstract}
Effect of pollen dispersion in corn seed production, in Texcoco, México. In order to find out how the pollen of corn is dispersed in a seed production field without isolation, and the implications on genetic quality of the seed, a field test was established (189 m long by $42.5 \mathrm{~m}$ wide) during Spring-Summer season of 1992 in Texcoco, Mexico. Three corn populations with contrasting endosperm composition were planted: two of normal field corn and other of sweet corn. The sweet corn variety was surrounded by three rows of the normal corn varieties. The variables measured were plant and ear length, male and female flowering period, percent of normal grain as indicator of the cross of the normal type varieties over the sweet type. Lack of physical isolation and coincidence of flowering periods favored crossing of the varieties. Pollen dispersion and contamination within the field followed a normal distribution; there were variations in the distance and level of contamination due to wind direction and vigor differences of contaminant plants. The lowest grade of contamination was $1 \%$ in the central part of the field. According to the response surface obtained, 25 border rows are recommended to obtain seed free of contamination.
\end{abstract}

Key words: Seed purity, pollen dispersion, border rows, outcrossing.

Palabras clave: Pureza de semilla, surcos borderos, dispersión de polen, cruzamiento.

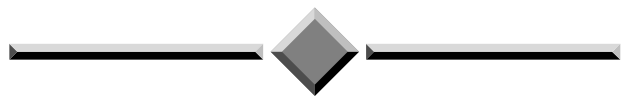

1 Recibido: 1 de octubre, 2009. Aceptado: 22 de noviembre, 2010. Este trabajo contiene parte de la información obtenida en la tesis de Maestro en Ciencias presentada por el primer autor al Colegio de Postgraduados en Ciencias Agrícolas, México.

2 Colegio de Postgraduados. Campus Puebla. Km. 125.5 Carr. Fed. Méx.-Pue., Santiago Momoxpan. San Pedro Cholula, Puebla. C.P. 72760. México. Teléfono: (222) 2851442, extensión 2074. Fax: (222) 2851444. enriqueortiz@ colpos.mx

3 Colegio de Postgraduados. Campus Montecillo. Km. 36.5 Carr. México-Texcoco, C.P. 56230 Montecillo, Estado de México. México. carballo@colpos.mx,amunozo@colpos.mx, felixgc@colpos.mx 


\section{INTRODUCCIÓN}

El uso de variedades mejoradas es una forma de incrementar la producción de maíz. Para ello, las semillas destinadas a la siembra deben llevar fielmente todas las características obtenidas por el fitomejorador. El maíz es una planta alógama, monoíca y con polinización típicamente anemófila. La polinización cruzada se favorece debido a que el viento y la gravedad son los agentes que transportan el grano de polen de la espiga al estigma (Bartolini 1990), lo que origina la posibilidad de polinización a grandes distancias. En el caso del maíz se han reportado cruzamientos a $200 \mathrm{~m}$ (Garcia et al. 1998 y Luna et al. 2001). La producción abundante de polen queda evidente con los reportes de Wesgate et al. (2003) quien menciona que una planta produce 4,5 x $10^{6}$ granos de polen por un lapso de cinco o seis días, en tanto que Kiesselbach (1980) calculó que se producen 25000 granos de polen para cada grano en una mazorca común.

En la producción de semilla de maíz se dificulta el control de la identidad genética, debido a que existe la posibilidad de polinización cruzada con materiales no deseados. Existen diversas recomendaciones para aislar los lotes de multiplicación de semillas de otros campos con maíz y reducir la contaminación por polen no deseado. Las recomendaciones más comunes son el aislamiento por distancia, por tiempo y con surcos borderos (Cowan 1972, Craig 1977). En el caso de México, las normas para la producción de semilla señalan que un lote de producción debe estar aislado $300 \mathrm{~m}$ en la producción de una variedad de polinización libre en la categoría certificada y $200 \mathrm{~m}$ si se trata de un lote de producción de híbridos (SNICS 1975). Sin embargo, en las regiones productoras de maíz no es posible obtener aislamiento como se establecen en las normas. En México, por sus condiciones orográficas, climáticas y étnicas existe una gran diversidad de nichos ecológicos, en donde es necesario generar localmente las variedades y producir del mismo modo la semilla requerida. Sin embargo, en la producción de semilla en estos nichos ecológicos, se limita de lograr un aislamiento por distancia de acuerdo a las normas de producción debido a que son zonas productoras de maíz.

En la actualidad en México, hay pocos estudios realizados respecto a las distancias de aislamiento de los lotes de producción de semilla que aseguren una buena calidad genética; se desconoce la cantidad de contaminación que puede ocurrir en lotes que no cumplen con las distancias de aislamiento establecidas en las normas, y las prácticas alternativas tendientes a reducir la contaminación por polen extraño.

Adicionalmente, es de interés actual conocer cómo se distribuye el polen para reducir riesgos de contaminación de materiales nativos con materiales transgénicos (Mercer y Wainwright 2008). Entre los trabajos en los que se ha intentado medir la distancia a la cual el polen puede transportarse y lograr la fecundación, se pueden mencionar los de Jones y Newell (1946), Bateman (1947), Raynor et al. (1972), Paterniani y Stort (1974), Goggi et al. (2006 y 2007) y Arrit et al. (2007); ellos concluyeron que la máxima cantidad de polen y cruzamiento se encuentra entre los 10 y $20 \mathrm{~m}$ de la fuente de origen de polen contaminante, y que los niveles de cruzamiento disminuyen considerablemente conforme se aleja de ella; sin embargo, reportan la existencia de cruzamiento con polen a $60,100,200$ y $250 \mathrm{~m}$.

Otros trabajos, como los de Garcia et al. (1998), Jemison y Vayda (2001), Luna et al.( 2001), Ma et al. (2004), Stevens et al. (2004), Goggi et al. (2006) y Messeguer et al. (2006), indican que la cantidad de polinización cruzada a grandes distancias es menor a $1 \%$, por lo cual sugieren la posibilidad de aceptar los lotes de producción de semilla con problemas de aislamiento por distancia si se considera además de la distancia, el aislamiento por tiempo y con surcos borderos.

Con base en lo anterior y partiendo de la necesidad de producir semilla a pequeña escala y en regiones productoras de maíz, en donde es difícil localizar zonas aisladas de polen de maíz, se hizo la presente investigación con el objetivo de determinar la dispersión del polen de maíz y su efecto en la calidad genética de la semilla.

\section{MATERIALES Y MÉTODOS}

El material genético utilizado fue de tres poblaciones de maíz contrastantes en color y composición del endospermo. Dos de ellas se usaron como contaminadoras, con el objeto de tener variación en la cantidad de polen producido, altura y vigor de planta. La primera fue la Colecta $1935^{4}$, de aleurona morada, endospermo cristalino y con 86 días a floración femenina. La

\footnotetext{
4 Proporcionada por el Dr. Abel Muñoz O., investigador del Colegio de Postgraduados.
} 
segunda fue la Cacahuacintle- $1^{5}$ una variedad de maíz de la raza Cacahuacintle, con endospermo blanco y harinoso, con 75 días a la floración femenina. Además, la variedad Dulce- $1^{6}$, con color de semilla amarillo y blanco, endospermo rugoso y duro, con 75 días a floración femenina, que se utilizó para cuantificar la contaminación genética.

El marcador genético que se utilizó para identificar la polinización cruzada entre poblaciones fue la característica de endospermo dulce, la cual se reconoce fácilmente por la apariencia vítrea y arrugada de la semilla cuando está madura. El maíz dulce difiere del normal solamente por un gene recesivo $(s u)$ y se manifiesta cuando está en condición homocigótica ( $s u s u$ ); en cambio, si se encuentra en condición homocigótica dominante o heterocigótica, da origen al endospermo normal. Dado que estos genes exhiben efecto de xenia, el cruzamiento entre variedades es detectado en la primera generación.

El experimento se condujo en terrenos del Campo Experimental San Juan de la Universidad Autónoma Chapingo, que se encuentra en San Dieguito, Texcoco, Estado de México (19³0’ N, 98 51' O, 2250 m de altitud). El clima es del tipo $\mathrm{Cb}(\mathrm{Wo})(\mathrm{W})\left(\mathrm{i}^{\prime}\right) \mathrm{g}$, que corresponde a un templado subhúmedo con lluvias en verano, una época seca en invierno y poca oscilación térmica entre 5 y $7^{\circ} \mathrm{C}$, la temperatura media anual es de $15,5^{\circ} \mathrm{C}$, siendo mayo el mes más caliente y enero el mes más frío. La precipitación media anual es de 644 mm (García 1981). Los vientos dominantes en otoño es la dirección noroeste y la velocidad promedio es de $2 \mathrm{~m} / \mathrm{s}$. La parcela experimental consistió en un lote aislado de $189 \mathrm{~m}$ de longitud y 42,4 $\mathrm{m}$ de ancho. Los surcos se hicieron en dirección norte-sur, separados a $80 \mathrm{~cm}$, siguiendo la longitud del terreno. La disposición de las variedades en el lote fue la siguiente: el maíz azul se sembró en los tres primeros surcos del lado oriente y en el primer metro de la cabecera norte, el maíz cacahuacintle se dispuso en los tres primeros surcos del lado poniente y en los dos primeros metros de la cabecera sur. El maíz dulce se colocó en el centro del lote, en los 47 surcos restantes, de esta forma los maíces de endospermo normal quedaron rodeando al maíz de endospermo dulce.

\footnotetext{
5 Proporcionada por el Dr. Aquiles Carballo C., investigador del Colegio de Postgraduados.

6 Proporcionada por el Dr. Fernando Castillo, investigador del Colegio de Postgraduados.
}

La siembra se hizo bajo riego y en fechas diferentes para hacer coincidir las floraciones. El maíz azul se sembró el 20 de mayo de 1992 y 10 días después las variedades restantes. Se empleó una densidad de población de 30 y 50 mil plantas por hectárea para las variedades Azul y Cacahuacintle, respectivamente, para tener variación en la cantidad de polen producido por las de endospermo normal. En la de endospermo dulce se usaron 42 mil plantas por hectárea.

Como el objetivo fue evaluar la dispersión del polen de las poblaciones de grano normal sobre la de endospermo dulce, no se usó ningún diseño experimental, sino que se muestreó todo la porción del lote con la variedad con endospermo dulce. En la cosecha, el lote conteniendo el maíz dulce se dividió en 888 parcelas de dos surcos de cinco metros de largo, excepto las adyacentes al maíz cacahuacintle, las cuales fueron de un surco. Las parcelas se identificaron con número de hilera y de columna. Las columnas se formaron perpendiculares al sentido de los surcos y se numeraron de norte a sur del uno al 37. Las hileras fueron paralelas a los surcos y se numeraron del uno al 24 en dirección oriente-poniente.

\section{Variables evaluadas}

Para describir las características de planta y de floración de las poblaciones estudiadas se tomó el promedio de altura de planta y de mazorca, días a floración media masculina y femenina. En una muestra de 100 plantas de cada población se determinó: altura de planta, midiéndola en centímetros desde el ras del suelo hasta la base de la espiga; altura de mazorca, evaluándola en centímetros desde el ras del suelo hasta el nudo en que estaba insertada la mazorca superior; periodo de la floración masculina, considerado como el número de plantas esparciendo polen; comportamiento de la floración femenina, que es el número de plantas que habían emitido sus estigmas. También se estimaron los días a floración media masculina, considerado como el número de días después de la siembra hasta cuando el 50\% de la población presentó antesis; y los días a floración media femenina, considerado como el número de días después de la siembra hasta cuando el 50\% de la población presentó floración masculina. La variable Porciento de Grano Normal (PORGRANO) únicamente se evaluó en la variedad dulce. En cada una de las parcelas de maíz dulce se cosecharon al menos 18 plantas 
y se desgranaron; las semillas así obtenidas se homogeneizaron y posteriormente se tomó al azar una muestra de 500 semillas. En esta muestra, se determinó el número de granos con endospermo dulce y normal, para así obtener el porcentaje de grano normal por parcela. Se hizo un análisis gráfico de los resultados, formando curvas de igual respuesta o isocuantas de los niveles de contaminación presentados en el lote de estudio.

\section{Análisis por superficie de respuesta}

Cochran y Cox (1965) comentan que cuando los factores o variables independientes representan cambios cuantitativos, la respuesta está en función de los niveles de los factores de acuerdo al modelo siguiente:

$$
\mathrm{Y}_{\mathrm{u}}=f\left(\mathrm{x}_{\mathrm{iu}}, \mathrm{x}_{2 \mathrm{u}}, \ldots, \mathrm{x}_{\mathrm{ku}}\right)+\mathrm{e}_{\mathrm{u}}
$$

donde: $f=$ función llamada superficie de respuesta; $\mathrm{u}=1,2, . . \mathrm{N} ; \mathrm{X}_{\mathrm{iu}}=$ representa el i-ésimo factor en la u-esima observación y $\mathrm{e}_{\mathrm{u}}=$ el error experimental de u-ésima observación. Cuando se conoce la forma matemática de la $f$, la función puede aproximarse satisfactoriamente dentro de la región experimental por un polinomio de las variables $\mathrm{X}_{\mathrm{iu}}$. La función $f$, así obtenida, da un resumen completo de los resultados del experimento y permite predecir y caracterizar las respuestas de los valores de las $\mathrm{X}_{\mathrm{iu}}$. En este sentido, con la finalidad de estudiar cómo se comporta la contaminación en función de las distancias a la fuente de ésta, los datos se ajustaron a un polinomio de segundo orden. Con la técnica de regresión lineal múltiple se produjeron los estimadores de los coeficientes de regresión y posteriormente una función estimada. El modelo utilizado fue el siguiente:

$$
\begin{gathered}
\mathrm{y}_{\mathrm{i}}=\beta_{0}+\beta_{1} \mathrm{COL}+\beta_{2} \mathrm{HIL}+\beta_{3} \mathrm{COL}^{2}+\beta_{4} \mathrm{HIL}^{2}+ \\
\beta_{5} \mathrm{COL} * \mathrm{HIL}+\mathrm{e}_{\mathrm{i}}
\end{gathered}
$$

donde: $\mathrm{y}_{\mathrm{i}}=$ representa el valor de la variable PORGRANO para la i-ésima parcela; $\beta_{0}=$ representa el valor de la ordenada al origen; $\beta_{1}$ y $\beta_{2}=$ son los coeficientes para los efectos lineales de columna (COL) e hilera (HIL) respectivamente; $\beta_{3}$ y $\beta_{4}=$ son los coeficientes de regresión para los efectos cuadráticos de columna $\left(\mathrm{COL}^{2}\right)$ e hilera $\left(\mathrm{HIL}^{2}\right)$, respectivamente; $\beta_{5}=$ es el coeficiente de regresión para la interacción columna por hilera $\left(\mathrm{COL}^{*} \mathrm{HIL}\right) ; \mathrm{y} \mathrm{e}_{\mathrm{i}}=$ error aleatorio debido al error de la i-ésima observación. Al modelo una vez ajustado se le practicó un análisis de varianza para probar si éste era adecuado, para explicar correctamente la respuesta en función de las variables dependientes estudiadas. Posteriormente, con la prueba de t de Student se procedió a probar la significancia de cada uno de los coeficientes que definieron la ecuación de respuesta para determinar si contribuían a explicar la función. El procesamiento electrónico de la información fue mediante el paquete estadístico SAS (SAS 2004). Para la interpretación de la superficie de respuesta se recurrió a una representación gráfica delineando contornos de igual respuesta o isocuantas las cuales forman elipses concéntricas.

\section{RESULTADOS Y DISCUSIÓN}

\section{Comportamiento de la floración}

Hubo coincidencia en la floración entre las variedades estudiadas. La variedad dulce y la cacahuacintle alcanzaron la floración media masculina a los 85 días y el maíz azul un día después. Los maíces dulce y azul tuvieron su floración media femenina a los 87 días y el cacahuacintle a los 90 días. Se observa a través del tiempo las frecuencias acumuladas del número de plantas con presencia de estigmas receptivos de la variedad dulce y con espigas en antesis de las poblaciones azul y blanca; aquí se observa que los periodos de floración masculina de los maíces azul y blanco cubrieron perfectamente el periodo de floración femenina del maíz dulce (Figura 1).

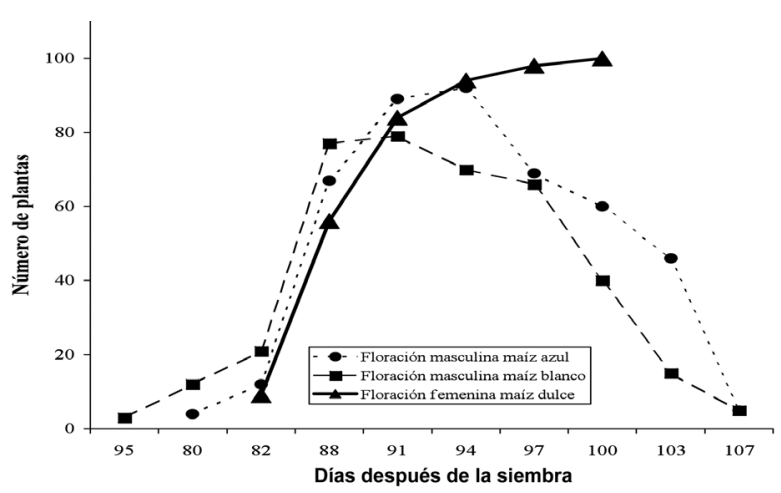

Figura 1. Comportamiento de la floración masculina de los maíces de endospermo normal y la floración femenina del maíz dulce estudiados en Texcoco, Estado de México, México. 1992. 


\section{Altura de planta y mazorca}

Una mayor altura de planta puede favorecer la dispersión del polen. Entre mayor sea la diferencia con la altura de jilote puede facilitar la polinización. La variedad cacahuacintle fue la de mayor altura con $176 \mathrm{~cm}$, le sigue la azul y la dulce con 153,5 y 143,1 $\mathrm{cm}$. La altura de jilote fue menos variable; el valor más alto fue para el maíz azul con $75,8 \mathrm{~cm}$ posteriormente el cacahuacintle y el dulce con 71,1 y $69,2 \mathrm{~cm}$ respectivamente.

\section{Dispersión del polen dentro del lote}

Se observa que el mayor grado de contaminación $(38,1 \%)$ se presentó en la hilera 24 , la cual era la más próxima al maíz cacahuacintle del lado poniente, y a medida que se aleja de la fuente el porcentaje de cruzamiento se redujo. Después de la hilera 21 (siete surcos) un aumento en hileras no redujo notoriamente la contaminación. En el extremo oriente el comportamiento fue similar al observado en el lado poniente, pero los niveles de contaminación fueron menores, siendo solamente $9 \%$ en la hilera más próxima al maíz azul. La contaminación más baja se registró en el centro del lote $(2,3 \%)$ (Figura 2$)$. En promedio, la mayor cantidad de polinización cruzada se presentó en las cercanías de la fuente contaminante y conforme se incrementó la distancia la cantidad de semillas con endospermo normal se redujo paulatinamente. En maíz, este mismo fenómeno fue observado por Jones y Newell (1946), Hutchcroft (1959), Raynor et al.

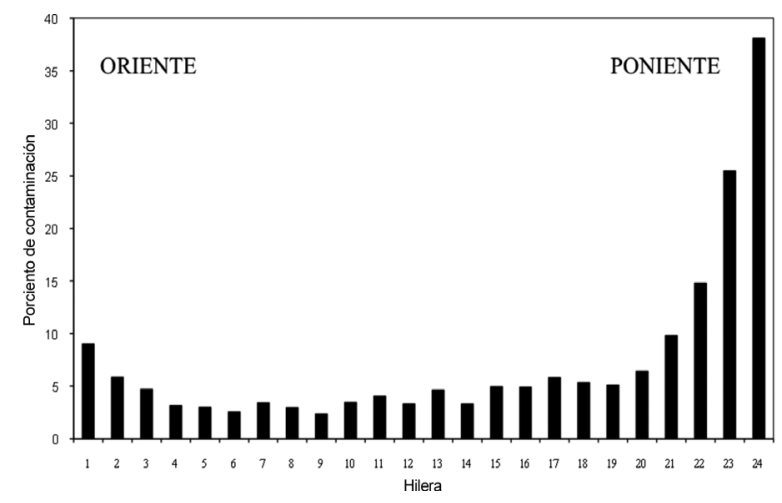

Figura 2. Promedios por hilera del porciento de grano normal obtenidos en el lote de estudio de dispersión de polen de maíz en Texcoco, Estado de México, México. 1992.
(1972), Bartolini (1990), Jarosz et al. (2003), Ma et al. (2004) y Goggi et al. (2006 y 2007). La variación en la cantidad de granos formados por el polen del maíz contaminante conforme se aleja de la fuente contaminante, se comportó como una distribución normal del mismo tipo que señalan Proctor y Yeo (1973) para el caso de las especies anemófilas. Esto es la frecuencia de cruzamiento es muy alta en la cercanía de la fuente contaminante y se reduce rápidamente al alejarse de éste. Este mismo comportamiento en la frecuencia de cruzamiento se observa en la Figura 2, en la porción poniente de la gráfica la frecuencia de cruzamiento es alta cerca de la fuente contaminante poniente. En la hilera 24 hubo casi $40 \%$ de cruzamiento y se reduce gradual y rápidamente hasta el nivel del $5 \%$ de la hilera 19, a sólo seis hileras de la fuente contaminante.

En los promedios por columna no fue notoria la reducción paulatina de la contaminación con el alejamiento del origen del polen extraño (Figura 3). El mayor nivel se presentó en las columnas 1 y 37 con 12,6 y $19 \%$ de PORGRANO respectivamente, las cuales corresponden a las parcelas más próximas a los contaminantes de los extremos norte y sur del lote respectivamente. En el resto de las columnas no hubo una tendencia definida; los niveles de PORGRANO fueron muy similares, ésto por que cada columna representa el promedio de cinco metros de cultivo en dirección a la fuente de polen extraño y, como se ve en el promedio de hileras, la reducción mas drástica de la polinización cruzada es en los primeros cuatro metros de la fuente de polen (Figura 2).

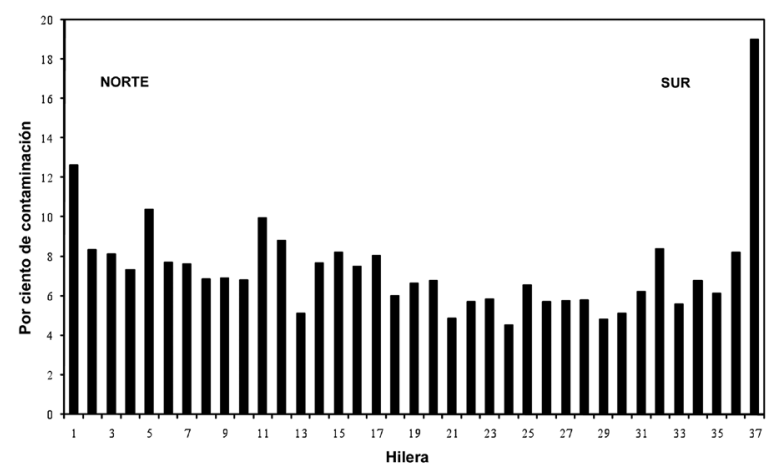

Figura 3. Promedios por columna del porciento de grano normal obtenidos en el lote de estudio de dispersión de polen de maíz en Texcoco, Estado de México. México. 1992. 
Entre las variedades usadas como marcadores no hubo homogeneidad en el grado de dispersión de polen y polinización cruzada. El maíz blanco presentó los mayores grados de contaminación tanto en la primera columna como en la primera hilera, así como también presentó niveles altos de contaminación a mayores distancias que el maíz azul. Esta diferencia puede deberse a varios factores que facilitaron la dispersión del polen del maíz blanco como son: mayor altura y densidad de planta. El mayor número de plantas del maíz blanco originó mayor producción de polen y, por lo tanto, incrementó la probabilidad de fecundar los estigmas del maíz dulce.

En los contornos con igual porcentaje de contaminación (Figura 4) se observó que en general el mayor nivel se presentó en los cuatro extremos del lote en las proximidades de la fuente de contaminación. Posteriormente, los niveles de contaminación se redujeron

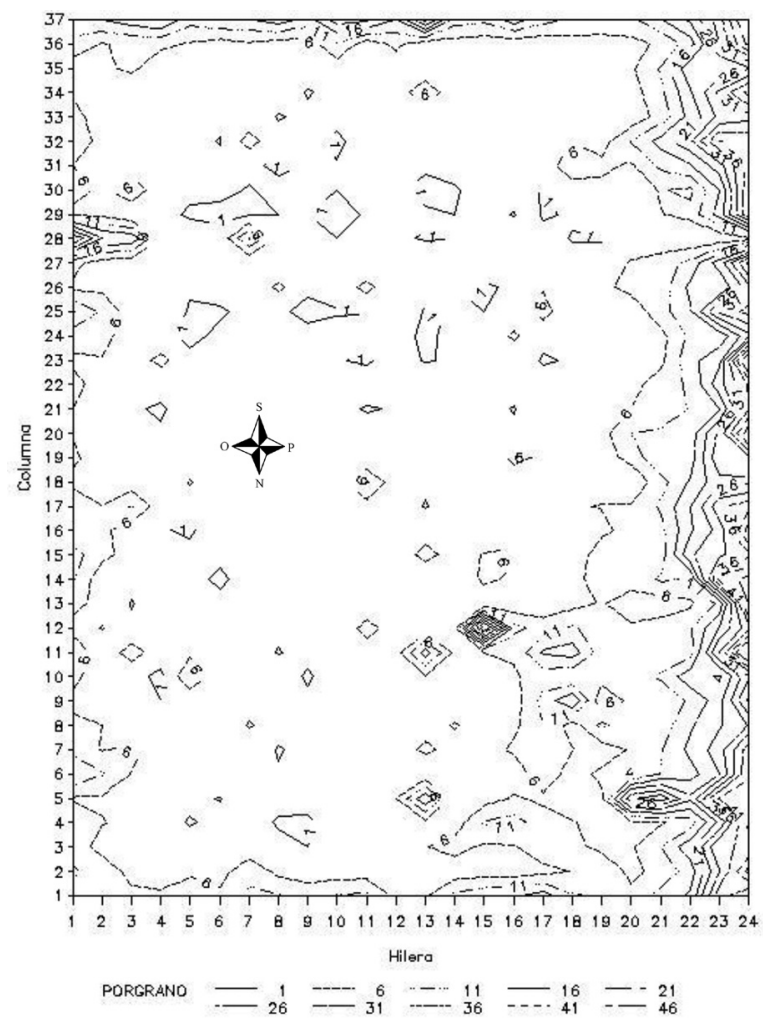

Figura 4. Contornos con igual porcentaje de grano normal dentro del lote de estudio de dispersión de polen en Texcoco, Estado de México, México. 1992. paulatinamente hacia el centro del lote; pero no en la misma magnitud.

Comparando los cuatro lados, los mayores niveles se observan en los extremos sur y poniente, en donde la fuente contaminante era el maíz blanco. Particularmente en el lado poniente se registraron los niveles más elevados de contaminación $(46 \%)$ en la hilera 24. Los niveles de uno por ciento de PORGRANO se localizaron únicamente en la parte central del lado sur. La isocuanta de $6 \%$ se extiende abarcando la parte central del lote, no obstante aun dentro de esta zona hay puntos aislados que sobrepasan este porcentaje de contaminación, como en las hileras y columnas 7-28, 13-5, 15-12 y 13-11. Esta variación en la dispersión del polen puede deberse, aunque en este trabajo no se tomaron medidas de velocidad y dirección del viento, a las diferentes direcciones que toma éste durante el período de floración, o a turbulencias ocasionales que transportaron el polen más lejos del origen en determinadas direcciones, tal como lo señalan Bateman (1947), Paterniani y Stort (1974), Ma et al. (2004), Goggi et al. (2006 y 2007) y Arritt et al. (2007).

La reducción drástica en la cantidad de polen que logra fecundar un estigma del maíz dulce al alejarse de la fuente contaminante puede ser ocasionada por la distancia física al origen. El polen de maíz es grande comparado con otras especies anemófilas similares, el maíz tiene en promedio 90-100 $\mu$ de diámetro (Raynor et al. 1972 y Pfahler 1978), este tamaño origina que la cantidad de polen en la atmósfera se reduzca rápidamente. La reducción drástica en la cantidad de polen atmosférico al alejarse del origen del polen ha sido reportada por diversos investigadores (Bateman 1947, Raynor et al. 1972, Bartolini 1990, Jarosz et al. 2003. La reducción de polen puede explicar el menor porcentaje de cruzamiento al alejarse de la fuente de polen (Ma et al. (2004). Goggi et al. (2006 y 2007) reportan que a $100 \mathrm{~m}$ el cruzamiento decrece al $0,05 \%$ o menos.

\section{Superficie de respuesta}

Se ajustaron los resultados al modelo propuesto. En el análisis de varianza de la ecuación de regresión obtenida, el modelo utilizado mostró ser altamente significativo $(\mathrm{p}<0,01)$, lo que indicó que al menos uno de los $\beta_{\mathrm{j}}$, con $\mathrm{j}=1 \ldots . .5$, es diferente de cero y que el modelo usado explicó bien la respuesta en función de las 
variables estudiadas. Con este antecedente se procedió a determinar la significancia de cada uno de los parámetros que definen la ecuación de respuesta (Cuadro 1). Todos los estimadores resultaron ser significativos $(\mathrm{P}<0,01)$; por lo tanto, contribuyen a explicar la función. Por lo anterior, la ecuación final de respuesta es la siguiente:

$$
\begin{gathered}
\mathrm{y}=14,386-0,447 \mathrm{COL}-2,254 \mathrm{HIL}+0,013 \mathrm{COL}^{2}+ \\
0,123 \mathrm{HIL}^{2}-0,007 \mathrm{COL} * \mathrm{HIL}
\end{gathered}
$$

En la ecuación cuadrática (Figura 5) se comprobó que los mayores niveles de contaminación están en las proximidades de la fuente contaminante y existió una reducción paulatina conforme se alejó de ella. De acuerdo a los resultados, el polen del maíz blanco se dispersó en mayor cantidad y distancia que el del maíz azul, en la primera hilera más próxima del maíz blanco se tiene $22 \%$ de contaminación mientras que del lado azul apenas alcanzó 8\%. También, la distancia necesaria para reducir la contaminación a $0 \%$ fue mayor en el lado del maíz blanco que del lado del maíz azul; en el primero fueron 25 surcos y en el segundo 14. La cantidad de polen normal que fecunda un estigma del maíz dulce se reduce rápidamente a poca distancia del origen del polen, pero a medida que se aleja es necesario incrementar la distancia para reducir el cruzamiento en una unidad; por ejemplo, para pasar de 22 a $20 \%$ en el

Cuadro 1. Prueba de t de Student para los estimadores de los coeficientes del modelo de superficie de respuesta obtenidos para estimar la contaminación en función de las distancias a la fuente de contaminación en el lote de estudio de dispersión de polen en Texcoco, Estado de México, México. 1992.

\begin{tabular}{cccc}
\hline Parámetro & Estimador & \multicolumn{1}{c}{ Tc } & Pr> T \\
\hline$\beta_{0}$ & 14,386 & 12,64 & 0,0001 \\
$\beta_{1}$ & $-0,447$ & $-5,12$ & 0,0001 \\
$\beta_{2}$ & $-2,254$ & $-16,58$ & 0,0001 \\
$\beta_{3}$ & 0,013 & 6,56 & 0,0001 \\
$\beta_{4}$ & 0,123 & 25,27 & 0,0001 \\
$\beta_{5}$ & 0,007 & $-2,67$ & 0,0078 \\
\hline
\end{tabular}

$\beta_{0}=$ representa el valor de la ordenada al origen; $\beta_{1}$ y $\beta_{2}=$ son los coeficientes para los efectos lineales de columna e hilera respectivamente; $\beta_{3}$ y $\beta_{4}=$ son los coeficientes de regresión para los efectos cuadráticos de columna e hilera, respectivamente; $\beta_{5}=$ es el coeficiente de regresión para la interacción columna por hilera. $\mathrm{Tc}=$ es el valor de $\mathrm{t}$ de Student calculado, $\mathrm{Pr}>\mathrm{T}=$ probabilidad.

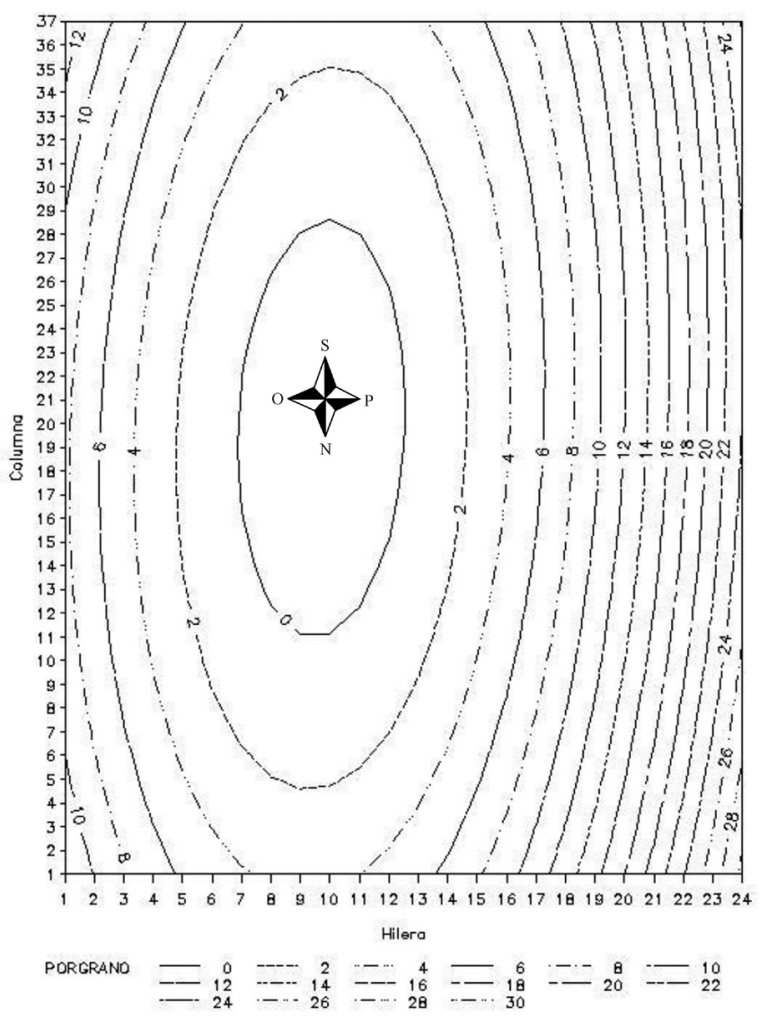

Figura 5. Contornos de igual porcentaje de grano normal según el modelo de respuesta ajustado para estimar la contaminación en función de las distancias a la fuente de contaminación en el lote de estudio de dispersión de polen. Texcoco, Estado de México, México. 1992.

lado poniente se necesitó un surco aproximadamente, mientras que para pasar de 2 a $0 \%$ fueron necesarios tres surcos. Según esta ecuación se localiza una zona de $0 \%$ de PORGRANO a 45 metros de la orilla sur, 60 metros de la orilla norte, 25 surcos de la poniente y 14 surcos de la oriente. Son mayores las distancias de las orillas norte y sur debido a que hay un efecto conjunto de tres fuentes de contaminación. Para un PORGRANO de $2 \%$ el área aumenta notoriamente, localizándose a $15 \mathrm{~m}$ del lado sur, 25 metros del norte, 10 surcos del oriente y 21 surcos del poniente.

En general el modelo estimado de superficie de respuesta permitió describir la influencia de la distancia a la fuente de contaminación y la del número de surcos borderos sobre el nivel de la misma; además se pueden realizar inferencias sobre el comportamiento 
del nivel de contaminación dentro del lote y predecir los niveles de los factores estudiados para los cuales la respuesta es mínima.

\section{Aislamiento obtenido}

Las condiciones que se simularon en el ensayo son los extremos que se pueden presentar en campo; esto es, sin aislamiento por distancia en las cuatro direcciones y completa sincronización en las floraciones. Aunque solamente fueron tres surcos del maíz contaminante, es una buena simulación de lo que pudiera acontecer en campo.

En general, en todo el lote hubo polinización por el maíz de endospermo normal. El valor mínimo de contaminación fue de uno por ciento, aunque este ocurrió en un área muy reducida en la parte central del lote. Considerando que el ancho del terreno que contenía al maíz dulce era de 37,6 m y que en ambos extremos habían tres surcos de un maíz contaminante, cabe resaltar que se logró un aislamiento en la parte central de casi 2,3\%, después de aproximadamente trece surcos $(10,4 \mathrm{~m})$ del lado poniente y ocho surcos $(6,4 \mathrm{~m})$ del lado oriente. Sin embargo, las normas para la producción de semillas certificadas permiten un máximo del uno por ciento de contaminación (SNICS 1975). Los resultados anteriores concuerdan con otros investigadores que plantean la utilización de surcos borderos para la reducción de la polinización. Sin embargo, el número de surcos borderos necesarios para reducir el nivel de polinización cruzada a uno por ciento es diferente. Ma et al. (2004) reportan que es necesario la remoción del equivalente a $30 \mathrm{~m}$ de terreno con plantas y Messeguer et al. (2006) concluyen que es necesario $20 \mathrm{~m}$ de cultivo. Goggi et al. (2006) encontraron que el cruzamiento no deseado puede reducirse colocando alrededor del lote una alta densidad de polen deseado. Las discrepancias en la cantidad de surcos borderos pueden deberse a las diferencias en la dirección y velocidad de los vientos que se presentaron en la época de floración, y a diferencias entre las variedades usadas en cada caso (Ma et al. 2004, Messeguer et al. 2006, Goggi et al. (2006 y 2007).

La ecuación de superficie de respuesta estimó que en las condiciones en que se realizó el ensayo es posible obtener una zona sin contaminación de $896 \mathrm{~m}^{2}$ en la parte central del lote, esta zona representa $12,2 \%$ del total de la superficie aprovechable. Para asegurar este nivel de calidad es necesario dejar 14 y 25 surcos de bordo en el lado oriente y poniente, respectivamente. El resto del lote presenta un nivel de contaminación inaceptable para la comercialización de semillas.

Todo lo anterior advierte que para cada lote de producción de semillas de maíz se deben tomar en cuenta varios aspectos para considerarlo como un lote aislado, entre los que destaca el aislamiento por distancia, el aislamiento por fecha y la utilización de surcos borderos. Es posible que manejando todos ellos, se logre una aceptable calidad genética de las semillas producidas, aun en las regiones donde sea difícil localizar lotes aislados únicamente por distancia.

La dispersión del polen de los maíces utilizados como fuente de contaminación dentro del lote de estudio siguió una distribución normal. La cantidad de contaminación se redujo rápidamente en los primeros ocho surcos de la fuente contaminante; no obstante, a medida que se aleja es necesario incrementar la distancia para reducir la contaminación en una unidad porcentual. La dispersión del polen no fue homogénea dentro del lote, existiendo variaciones en los niveles y distancias de contaminación debidas posiblemente a diferencias en la densidad de población, en el vigor de las plantas y en el polen producido por cada población usada como contaminante. En todo el lote de maíz dulce hubo contaminación, siendo la menor la de $1 \%$ que se presentó en el centro del lote. Las condiciones que facilitaron la contaminación dentro del lote fueron: la inexistencia de aislamiento por distancia y la coincidencia total de las floraciones. La ecuación estimada de superficie de respuesta, en las condiciones en que se realizó el ensayo, considera que para asegurar un nivel de contaminación de $0 \%$ en la parte central del lote se debe eliminar a 45 metros de la orilla sur, 60 metros de la norte, 25 de surcos del lado poniente y 14 surcos del oriente.

\section{LITERATURA CITADA}

Arritt, R; Clark, C; Goggi, SA; Lopez-Sanchez, H; Westgate, M; Riese, JM. 2007. Lagrangian numerical simulations of canopy air flow effects on maize pollen dispersal. Field Crops Research 102(2):151-162.

Bartolini, R. 1990. El maíz. Mundi-Prensa. Madrid, España. p. 2-25.

Bateman, AJ. 1947. Contamination of seed crops II. Wind pollination. Heredity 1:235-246. 
Cochran, WG; Cox, GM. 1965. Diseños experimentales. Trad. Centro de Estadística y Cálculo del C. P. Trillas. México. p. 372-415.

Cowan, JR. 1972. Seed certification. In Kozlowski, TT. ed. Seed Biology. Academic Press. USA. 3 v. p. 371- 391.

Craig, WF. 1977. Production of hybrid seed. In: Sprague, GF. ed. Corn and corn improvement. Agronomy 18. American Society of Agronomy. p. 671-719.

García, E. 1981. Modificaciones al sistema de clasificación climatica de Köppen; para adaptarlo a las condiciones de la República Mexicana. Dirección General de Publicaciones. UNAM. México, D.F. p. 132.

Garcia C, C; Figueroa M, J; Gomez L, R; Townsend, R; Schoper, J. 1998. Pollen control during transgenic hybrid maize development in Mexico. Crop Science 38:1597-1602.

Goggi, SA; Lopez-Sanchez, H; Caragea, P; Westgate, M; Arritt, R; Clark, C. 2007. Gene flow in maize fields with different local pollen densities. International Journal of Biometeorology 51:493-503.

Goggi, SA; Caragea, P; Lopez-Sanchez, H; Westgate, M; Arritt, R; Clark, C. 2006. Statistical analysis of outcrossing between adjacent maize grain production fields. Field Crops Research 99:147-157.

Hutchcroft, CD. 1959. Contamination in seed fields of corn resulting from incomplete datasseling. Agronomy Journal 51:267-271.

Jarosz, N; Loubet, B; Durand, B; McCartney, A; Foueillassar, X; Huber, L. 2003. Field measurements of airborne concentration and deposition rate of maize pollen. Agricultural and Forest Meteorology 119:37-51.

Jemison, JM; Vayda, ME. 2001. Cross pollination from genetically engineered corn: wind transport and seed source. AgBioForum 4(2):87-92.

Jones, MD; Newell, LC. 1946. Pollination cycles and pollen dispersal in relation to grass improvement. Research. Bulletin 148. Agricultural Experiment Station of University of Nebraska. Lincoln, USA. 43 p.

Kiesselbach, TA. 1980. The structure and reproduction of corn. University of Nebraska Press. USA. p. 1-50.
Luna V, S; Figueroa M, J; Baltazar M, B; Gomez L, R; Townsend, R; Schoper, JB. 2001. Maize pollen longevity and distance isolation requirements for effective pollen control. Crop Science 41:15511557.

Ma, BL; Subedi, KD; Reid, LM. 2004. Extent of crossfertilization in maize by pollen from neighbouring transgenic hybrids. Crop Science 44:1273-1282.

Mercer, KL; Wainwright, JD. 2008. Gene flow from transgenic maize to landraces in Mexico. Agriculture, Ecosytems and Environment 123:109115.

Messeguer, J; Peñas, G; Ballester, J; Bas, M; Serra, J; Salvia, J; Palaudelmas, M; Melé, E. 2006. Pollen-mediated gene flow in maize in real situations of coexistence. Plant Biotechnology Journal 4(6):633-645.

Paterniani, E; Stort, AC. 1974. Effective maize pollen dispersal in the field. Euphytica 23:129-134.

Pfahler, PL. 1978. Biology of the maize male gametophyte. In Wlden, DB ed. Maize breeding and genetics. Wiley. New York, USA. p. 517-530.

Proctor, M; Yeo, P. 1973. The pollination of flowers. Willam Collins Sons. London, UK. p. 256-275.

Raynor, GS; Ogden, EC; Hayes, JV. 1972. Dispersion and deposition of corn pollen from experimental sources. Agronomy Journal 64:420-427.

SAS (Statistical Analysis System). 2004. User's guide. Version 9.1. SAS Institute Inc. Cary, NC, USA. p. 1731-1900.

SNICS (Servicio Nacional de Inspección y Certificación de Semillas). 1975. Normas para la certificación de semillas. Secretaría de Agricultura y Ganadería. México. p. 66-69.

Stevens, WE; Berberich, SA; Sheckell, PA; Wiltse, CC; Halsey, ME; Horak, MJ; Dunn, DJ. 2004. Optimizing pollen confinement in maize grown for regulated products. Crop Science 44:2146-2153.

Westgate, ME; Lizaso, J; Batchelor, W. 2003. Quantitative relationship between pollen-shed density and grain yield in maize. Crop Science 43:934-942. 